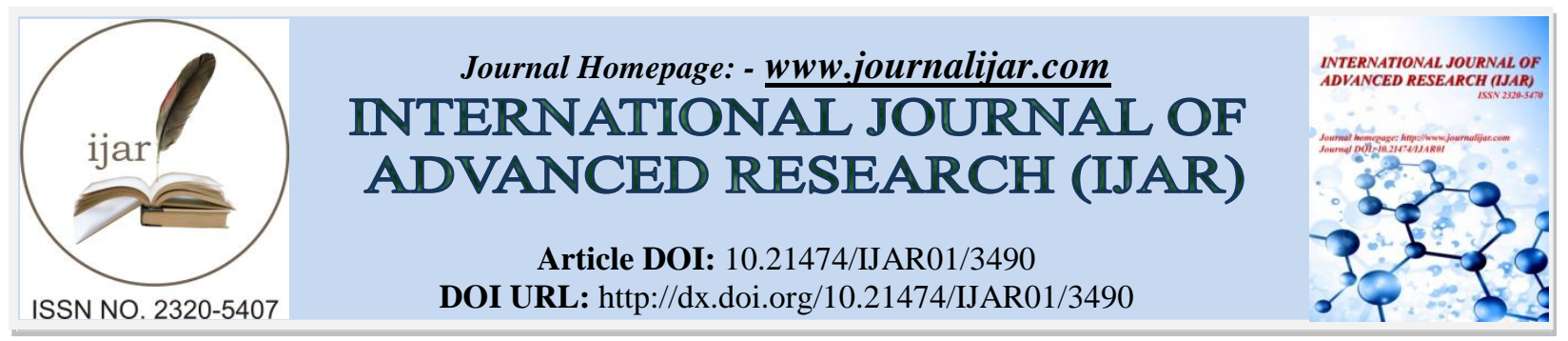

RESEARCH ARTICLE

\title{
THE USEFULNESS \& ACCURACY OF THE MODIFIED ALVARADO SCORE IN THE PREOPERATIVE DIAGNOSIS OF ACUTE APPENDICITIS.
}

\author{
Dr. Mounis Mahdi Salih.
}

M.B.CH.B, F.I.C.M.S, Senior in general surgery at AL-Yarmouk Teaching Hospital.

\section{Manuscript Info}

Manuscript History

Received: 10 January 2017

Final Accepted: 12 February 2017

Published: March 2017

\section{Abstract}

This prospective study was conducted to evaluate the usefulness \& accuracy of the modified Alvarado score in the preoperative diagnosis of acute appendicitis. The study included one hundred \& fifty patients of different age groups \& from both genders with provisional diagnosis of acute appendicitis presented to the emergency department of Al- Yarmouk Teaching Hospital over eighteen months period. All patients were evaluated on admission \& given specific scores according to the variables of Modified Alvarado Score. In adult male patients acute appendicitis was confirmed in (40) of (42) patients giving sensitivity rate of $(88.8 \%)$ \& specificity rate of $(91.6 \%)$, in adult female, it was confirmed in (25) of (28) patients with sensitivity rate of $(80.6 \%) \&$ specificity rate of $(89.6 \%)$, while in children acute appendicitis was confirmed in (7) of (9) patients giving a sensitivity rate of $(53.8 \%) \&$ specificity rate of $(75 \%)$. The overall sensitivity rate of Modified Alvarado Score was $80.9 \%$ \& its specificity was $88.5 \%$. Total negative appendicectomy rate was $15.2 \%$. The Modified Alvarado Score is simple, easy to apply \& cheap complementary aid for supporting the diagnosis of acute appendicitis especially for junior surgeons.

Copy Right, IJAR, 2017,. All rights reserved.

\section{Introduction:-}

The Alvarado Score was described in 1986. ${ }^{(1)} \&$ has been validated in adult surgical practice. ${ }^{(1)}$ The Modified Alvarado Score is a simplified modification of the original Alvarado Score after the omission of one of its parameters by Kalan et al. ${ }^{(2)}$ Since 1886 when the classical signs \& symptoms of acute appendicitis were first reported by Fitz. ${ }^{(1)}$ Acute appendicitis has remained the most common surgical emergency. ${ }^{(3)} \&$ surgery for acute appendicitis is the most frequent operation performed $(10 \%$ of all emergency abdominal operations). (5,6) Approximately 6- 7\% of population will suffer from acute appendicitis during their life time . ${ }^{(7)}$ With early diagnosis \& intervention the mortality rate from non complicated acute appendicitis is less than $0.1-0.6 \%$ when there is gangrene, \& $5 \%$ for perforated cases.$^{(8)}$ The diagnosis of acute appendicitis is purely based on history, clinical examination \& some simple laboratory investigations like white blood cells count. Recently the attempt to increase the diagnostic accuracy in acute appendicitis by imaging, laparoscopy \& even radioactive isotope imaging have been included, however these techniques involve additional costs $\&$ are either complex or not easily available when they are most needed. A negative appendicectomy rate of $20-40 \%$ has been reported in literatures $\&$ many surgeons would accept rate of $30 \%$ as inevitable ${ }^{(9)}$ The Modified Alvarado Score was designed to reduce the negative appendicectomy rate without increasing the morbidity \& mortality. ${ }^{(10)}$ At present many scoring systems for 
diagnosis of acute appendicitis are available \& the Modified Alvarado Score is one of them, \& is based on history, clinical examination \& simple laboratory tests, $\&$ is very easy to apply as shown below. ${ }^{\text {(Il) }}$

Score

The Modified Alvarado

SYMPTOM

A-Migratory right iliac fossa pain

B-Anorexia

C-Nausea \& vomiting

SIGNS

A-Tenderness in lower quadrant of abdomen

$\mathrm{B}-$ Rebound tenderness in right iliac fossa

C-Pyrexia equal or more than $37.5 \mathrm{c}^{0}$

3-Investigation

A-Leuococytosis

TOTAL SCORE
SCORE

1

1

1

2

1

1

2

9

The application of the score is based on the above variables ( 3 symptoms, 3 signs, 1 test). Patients with score 1- 4 are considered unlikely to have acute appendicitis, those with a score of 5- 6 have possible diagnosis of acute appendicitis not convincing enough to have urgent surgery, \& those with score 7- 9 are regarded as probable acute appendicitis \& underwent an appendicectomy.

Aim of the Study:-

1. To evaluate the usefulness \& the validity of Modified Alvarado Score in the preoperative diagnosis of acute appendicitis.

2. To evaluate the accuracy of Modified Alvarado Score in relation to differences in age group \& gender.

3. To compare the results of negative appendicectomy rate in those who were evaluated by Modified Alvarado Score with clinical literature figures.

Patients \& Methods:-

This study was conducted on (150) patients admitted from the emergency department at Al- Yarmouk teaching Hospital with the clinical diagnosis of acute appendicitis during the period from June 2004 to December 2005. They were (79) males \& (71) females with their age ranging from 8- 65 years, however, 21 of them were children (age < 12 years). All included patients were prospectively evaluated using the Modified Alvarado Score to determine whether they had acute appendicitis or not.

Following admission, initial assessment $\&$ baseline investigations, a specially designed forma was filled for each patient. This forma had general information about the patient plus seven variables based on the Modified Alvarado Score \& information regarding the final outcome of each patient which was filled later on.

The admission of the suspected cases, evaluation \& the decision to operate was made by the surgical, on call team, on the bases of one weekly duty for the eighteen months during which this study was conducted.

The sum of all variables were calculated for each patient $\&$ based on the results, patients were divided into: Group one:- With score of $>7$ (emergency surgery group).

They were (79) patients comprised of (42) male, (28) female \& (9) children, these patients were prepared \& underwent appendicectomy.

Group two. With score of (5- 6) (observational group). They were (71) patients comprised of (27) male, (32) female $\& 12$ children. They were admitted \& kept under observation with frequent assessments \& re evaluations of the clinical data \& their score, every 4 - 6 hours. Abdominal ultrasonography was performed in 12 doubtful cases leading to the diagnosis of (2) cases of right ovarian cysts \& (2) cases of right ureteric stones, while the condition of (41) patients were improved as shown by the decrease in the intensity of the symptoms \& decrease in their score, \& they were discharged with instructions that they should come back if their symptoms persist or increase in intensity. However, (26) patients of this group underwent appendicectomy for either persistence or increase in their symptoms, or increase score on subsequent reevaluation. Any patient presented with score of $<(4)$ was not included in this 
study as they were considered unlikely to have acute appendicitis \& so they were discharged after initial symptomatic treatment with the instructions to come back if symptoms persist or increase in the intensity. Acute appendicitis was confirmed either peroperatively in those patients with macroscopical evidence of acute inflammation or by histopathological examination for those with macroscopically normal looking appendix

Results :- This study included (150) patients with clinical features suggestive of acute appendicitis. Among those patients (79) were male $(52.7 \%), \&(71)$ were female $(47.3 \%)$. The age of the patients included in this study range from 8 year to 65 year, The frequency distribution of the patients according to the results of Modified Alvarado Score demonstrate that the highest frequency was in those patients scored $5(40$ patients, $26.7 \%) \&$ the lowest frequency in patients scored 9 (20 patients, 13.3), However, from (150) patients (79) patients (52.6\%) had score of (7) or more,comprised of (42) adult male, (28) adult female \& (9) children, While (71) patients (47.4\%) had a score of less than (7), (5- 6), comprised of (27) adult male, (32) adult female \& (12) children. Another (41) patients with score of (5- 6) were discharged home as clinical improvement \& decreased score was shown in subsequent reevaluation, \& were instructed to come back if the symptoms persist or increase in intensity, while appendicectomy was performed in (26) patients with score of (5-6), they were (9) adult males, (10) adult females \& (7) children.

Acute appendicitis was confirmed in (17) patients (false-ve) comprised of (5) adult male, (6) adult female \& (6) children giving a specificity rate of $(91.6 \%),(89.6 \%)(75 \%)$ respectively. The overall sensitivity rate $\&$ specificity rate of Modified Alvarado Score were $\mathbf{( 8 0 . 9 \% ) ~ \& ~}(\mathbf{8 8 . 5 \%})$ respectively. The validity of Modified Alvarado Score for the (150) patients with provisional diagnosis of acute appendicitis. All patients with score of (7) or more ((79) patients) underwent appendicectomy. Acute appendicitis was confirmed in (72) patients (true +ve), among them (40) patients were adult male out of (42) giving sensitivity rate of $(88.8 \%)$, (25) adult female out of (28) giving sensitivity rate of $(80.6 \%) \&(7)$ children out of (9) giving sensitivity rate of (53.8). All patients with score of (5-6) (71 patients) were admitted for observation \& re evaluation. Abdominal ultrasonography was done for (12) patients leading to the diagnosis of (2) right ovarian cysts \& (2) ureteric stones, \& they were referred to the gynaecological \& urological departments respectively following clinical improvement. Another (41) patients with score of (5- 6) were discharged home as clinical improvement \& decreased score was shown in subsequent reevaluation, \& were instructed to come back if the symptoms persist or increase in intensity, while appendicectomy was performed in (26) patients with score of (5-6), they were (9) adult males, (10) adult females \& (7) children. Acute appendicitis was confirmed in (17) patients (false-ve) comprised of (5) adult male, (6) adult female \& (6) children giving a specificity rate of $(91.6 \%),(89.6 \%)(75 \%)$ respectively. Another (41) patients with score of (5- 6) were discharged home as clinical improvement $\&$ decreased score was shown in subsequent reevaluation, $\&$ were instructed to come back if the symptoms persist or increase in intensity, while appendicectomy was performed in (26) patients with score of (5-6), they were (9) adult males, (10) adult females \& (7) children. Acute appendicitis was confirmed in (17) patients (false-ve) comprised of (5) adult male, (6) adult female \& (6) children giving a specificity rate of $(91.6 \%),(89.6 \%)(75 \%)$ respectively The overall sensitivity rate \& specificity rate of Modified Alvarado Score were $(80.9 \%) \&(88.5 \%)$ respectivelyThe total number of patients who underwent appendicectomy was (105) patients (70\%) out of (150), among them acute appendicitis was confirmed in (89) patients (84.8) comprised of (72) patients with score of $>7$ (true +ve) \& (17) patients with score of $<7$ (false -ve), among them (8) had perforated appendices, (10) patients had gangrenous appendices \& (4) had appendicular mass, there was no operative findings of perforated, gangrenous appendices or appendicular mass in those patients with score $<7$. While normal appendix was found in (16) patients, (7) patients of them with score of $>7$ (false +ve) comprised of (2) adult male, (3)adult female $6 c$ (2) children. However, other surgical corrected pathology was found in (1) adult male with Meckel's diverticulitis \& (2) adult female one with ruptured right ovarian cysts \& the other with ruptured right tubal pregnancy. While in the remaining (4) patients no obvious other surgical pathology was identified. The other (9) patients with normal appendices were with score of $<7$ (true -ve) comprised of (4) adult male, (4) adult female $\&$ (1) child, \& other surgically corrected pathology was identified in (2) adult female only, one with ruptured right ovarian cyst \& one with right ovarian endometriotic (chocolate) cyst, while in the remaining (7) patients no obvious surgically corrected pathology was identified. So the total (-ve) appendicectomy rate was (15.2\%) \& the adult male, adult female \& children (-ve) appendicectomy rates were $(11.7 \%),(18.4 \%) \&(18.7 \%)$ respectively. The positive predictive value of the Modified Alvarado score was $91.1 \%$ \& for adult males, adult females \& children were $95.2 \%, 89.3 \%$ \& $77.8 \%$ respectively. 
Patients distribution with score $>7$

\begin{tabular}{|l|l|l|l|}
\hline Patients & Total no. & Score more 7 & $\%$ \\
\hline Adult male & 69 & 42 & 53.2 \\
\hline Adult female & 60 & 28 & 35.4 \\
\hline Children & 21 & 9 & 11.4 \\
\hline Total & 150 & 79 & 100 \\
\hline
\end{tabular}

Specificity of Modified Alvarado Score

\begin{tabular}{|l|l|l|l|l|l|}
\hline Patients & No. & Score & Operated & Appendicitis & Specificity \\
\hline Male & 69 & 27 & 9 & 5 & 91.6 \\
\hline Female & 60 & 32 & 10 & 6 & 89.6 \\
\hline Children & 21 & 12 & 7 & 6 & 75 \\
\hline Total & 150 & 71 & 26 & 17 & 88.5 \\
\hline
\end{tabular}

The sensitivity of Modified Alvarado Score

\begin{tabular}{|l|l|l|l|l|}
\hline & No. of patients & Score & Appendicitis & Sensitivity \\
\hline Male & 69 & 42 & 40 & 88.8 \\
\hline Female & 60 & 28 & 25 & 80.6 \\
\hline Children & 21 & 9 & 7 & 53.8 \\
\hline Total & 150 & 79 & 72 & 80.9 \\
\hline
\end{tabular}

\section{Specificity of Modified Alvarado Score}

\begin{tabular}{|l|l|l|l|l|l|}
\hline Patients & No. & Score & Operated & Appendicitis & Specificity \\
\hline Male & 69 & 27 & 9 & 5 & 91.6 \\
\hline Female & 60 & 32 & 10 & 6 & 89.6 \\
\hline Children & 21 & 12 & 7 & 6 & 75 \\
\hline Total & 150 & 71 & 26 & 17 & 88.5 \\
\hline
\end{tabular}

Results of application of Modified Alvarado Scor

\begin{tabular}{|l|l|l|l|}
\hline Item & Total No. & Score more 7 & Score $<7$ \\
\hline Total patients & 150 & 79 & 71 \\
\hline No surgery & 45 & 0 & 45 \\
\hline Surgery & 105 & 79 & 26 \\
\hline Acute appendicitis & 89 & 72 & 17 \\
\hline Normal appendix & 16 & 7 & 9 \\
\hline
\end{tabular}

The results of operative $\&$ histopathological

\begin{tabular}{|l|l|l|}
\hline Finding & No. of patients \% \\
\hline Inflamed acute appendicitis & 67 & 63.8 \\
\hline Acute appendicitis & 8 & 7.6 \\
\hline Perforated appendix & 10 & 9.6 \\
\hline Gangrenous appendix & 4 & 3.8 \\
\hline Appendicular mass & Normal appendix 1 \\
\hline & 2 & 1.9 \\
\hline Right ovarian cyst & 1 & 0.9 \\
\hline Meckel's diverticulitis & 1 & 0.9 \\
\hline Ruptured tubal pregnancy & 1 & 0.9 \\
\hline Endometriotic cyst & 11 & 10.6 \\
\hline No pathology & 105 & 100 \\
\hline Total operated on & & \\
\hline
\end{tabular}




\section{Discussion:-}

Acute appendicitis is undoubtedly, the most common surgical emergency, \& the diagnosis of acute appendicitis is essentially \& largely made on clinical grounds, but even then different possibilities are there.The prevalent age group in our study was from (11) to (30) years $(71.4 \%) \&$ the lowest rate were encountered in children $\&$ the elderly. These results are comparable with the results of a study done in Nineveh, Iraq, 1998 . $^{(12)}$ \& also with a study done in Pakistan at 2003. ${ }^{(13)}$ The frequency distribution of the patients included in our study according to Modified Alvarado Score demonstrate that the highest frequency was encountered with those who scored (6) (40 patients, $26.7 \%) \&$ the lowest frequency was in those scored (9) (20 patients, $13.3 \%)$. However, (79) patients (52.6\%) of the total (150) patients included in this study were with score of (7) or more \& (71) patients (47.4\%) were with score of less than (7) (5-6). These results are comparable to the results shown by a study done in Nineveh, Iraq, 1998. ${ }^{(12)} \&$ also with the results of a study done in Pakistan at 2003..$^{(13)}$

Initial assessment of the patients can be improved by clinical scoring system. ${ }^{(14,15)} \&$ in the past few years various scores have been developed to aid the diagnosis of acute appendicitis. ${ }^{(16,17,18)}$ However, most of them are complex $\&$ difficult to implement in clinical situation. ${ }^{(19,20,21)}$ Our findings demonstrate that Modified Alvarado Score carries results which may vary according to the age group (adult versus children) \& gender. For adult male patients with suspected acute appendicitis, the Modified Alvarado Score gives a sensitivity rate of $(88.9 \%)$ while in adult female $\&$ especially those at child bearing age Modified Alvarado Score gives a sensitivity rate of $(80.6 / 0)$ with a false (+ve) rate of (10.7). These findings are similar to the results of a study done in Nineveh, Iraq, $1998 \&$ also similar to the conclusions of a study done by Owen et of. ${ }^{(12)}$ However, they are not similar to the results of a study done in Southern Region, Kingdom of Saudi Arabia (sensitivity male 56.4\%, sensitivity female 48\%). Our study also revealed that Modified Alvarado Score is more helpful in adult male than adult female patients by showing lower (ve) appendicectomy rate $\&$ higher (+ve) predictive value in adult male as compared to adult female patients, as the (-ve) appendicectomy rate for adult male were $(11.7 \%)$ while, in adult female it was $(18.4 \%) \&$ the (+ve) predictive value were $(95.2 \%) \&(89.3 \%)$ for adult male \& female respectively. These results are comparable to a study done in Pakistan, 2003, also these results are supported by literature figures. ${ }^{(23,24,25)}$ Literatures show that if (-ve) appendicectomy rate is less than (10-15\%), then the surgeon is operating too few patients thus increasing the risk of complications. ${ }^{(26,27)}$ In children with acute appendicitis the sensitivity rate of Modified Alvarado Score in our study was very low $(53.8 \%) \&$ its specificity rate was (75\%) with a false (+ve) rate of more than (22\%), \& to be useful, a scoring system must be both sensitive \& specific. These findings are comparable to the results of a study done in Nineveh, Iraq, 1998 \& is also similar to the results of a study done by Macklin CP. ${ }^{(20)}$ The (+ve) predictive value for the children included in our study was (77.8\%). Studies evaluating the usefulness of Alvarado scoring system in paediatric age group show that it is accurate in children with (+ve) predictive value up to $(85.7 \%)$. ${ }^{(28,29,30)}$ The overall sensitivity \& specificity of our study were $(80.9 \%) \&(88.5 \%)$ respectively. These results arc comparable to the results of a study done in Nineveh, raq, 1998 \& is also comparable to the results of a study done by Fenyo G, but our results are not similar to the results of a study done in Southern Region, Kingdom Saudi Arabia. Our study revealed a total negative appendicectomy rate of $(15.2 \%)$ with a (-ve) appendicectomy rate of the adult male, adult female \& children of $(11.7 \%),(18.4 \%), \&(18.7 \%)$ respectively. These results are comparable to the results of a study done in Pakistan at $2003, \&$ are also similar to the results of a study done by Fenyo. ${ }^{(31)} \&$ is comparable to the figures shown in literature $(15.62 \%) .^{(32,33)}$ Our study showed a low incidence of perforation $(7.6 \%)$ which can be explained by the fact that perforation in acute appendicitis is clearly more common in children \& elderly people. ${ }^{(34,35)}$ The percentage of both of these groups is low in our study, as well as, the more accurate diagnosis depending on the application of Modified Alvarado Score. These results are comparable to the results of a study done in Nineveh, Iraq, 1998 \& is also comparable to the results of a study done in Pakistan at 2003.

\section{Conclusions \& Recommendation:-}

1. Modified Alvarado Score is a non invasive, safe diagnostic procedure which is simple, fast, reliable \& repeatable tool to apply in emergency departments to role in acute appendicitis. It allows observation \&critical reevaluation of the evolution of the clinical picture. We suggest its application in order to increase the diagnostic certainty of clinical examination in diagnosis of acute appendicitis especially by junior surgeons.

2. Modified Alvarado Score appeared to be effective in adult male patients, while in adult female patients specially those in child bearing age, additional investigations may be required, so we suggest the provision of (24) hours ultrasound facility to confirm or exclude the possibility of other pathologies in doubtful cases. In pediatric age group it was not successful enough to be useful complementary method in the diagnosis of suspected cases \& because our study included only a small number of children we suggest a prospective study 
to be conducted purely on patients within pediatric age group to assess precisely the validity of the Modified Alvarado Score in these cases.

3. Our study demonstrates comparable results of negative appendicectomy rate with that of literature figures, which signifies that the application of Modified Alvarado Score improves the diagnostic accuracy \& consequently reduces the negative exploration \& complication rates.

\section{References:-}

1. A A PmCt'Cal SCOre for lhe diagnosis of acute apnen'd icitis. Ann Emerg Med 1986; 15:557-564

2. H Rich AJ', Evaluation of the Modifted Alvarado SCore in diagnosis of acute appendicitis: a prospective study. Ann R. Coll. Surg. Eng. 1994, 76:418-19.

3. Y ft J.B. Acute appendicitis: US evaluation using grading compression. Radiology 1986; 158: 355-360.

4. Pearson R. H. Ultrasonography for diagnosing appendicitis. Br Med J. 1988; 297: 309-310.

5. Pal KM, Khan A. Appendicitis, a continuing challenge. J Pak Med Assoc 1998; 48: 189-92.

6. Kumar V, Cotran RS, Robbins SL. Appendix; In Robbin's Basic Pathology. 5th ed. London: W.B. Saunders $1992 ; 520$.

7. Wagner J.M. McKinney W.P. \& Carpenter J.L.: Does this patient have acute appendicitis. JAMMA Middle East. Vol. 276, P. 1589- 1594.

8. Butlor J. A. et al Evaluation of Tc $99 \mathrm{~m}$ leucocyte scan in the diagnosis of acute appendicitis. Journal of surgical research. Vol. 42, 1987, P. 575-579.

9. Kalan M. Talbot D, Cunliffe WJ, Rich AJ. Evaluation of the Modified Alvarado Score in the diagnosis of acute appendicitis: acute appendicitis a prospective study. Ann R Coll Surg 1994; 418-90. .

10. Alvarado A: A practical score for the early dtagnosts of acute

11. appendicitis. Am. Emerg. Med. 1986,15:55-65

12. Stephens PL, Mazzucco JJ. Comparison of ultrasound \& the Alvarado for \& diagnosis of acute appendicitis; Conn Med 1999; 63:137-40.

13. Issam Merdan Jebir; Evaluation of the Modified Alvarado Score in the diagnosis of acute appendicitis, A thesis supervised by Ass Prof Adnan Y. Abdul- Wahab 1998; page 29.

14. Ikramullah Khan, Ata ur Rehman; application of Alvarado scoring system in diagnosis of acute appendicitis, Department of surgery, Surg. Bunit, Khyber Teaching Hospital, Peshawar, Pakistan, Literature 2003.

15. Hoffman JO, Rasmussen O. Aids in \& diagnosis of acute appendicitis. Br J Surg 1989; 76: 774-9.

16. Owen T.D., Williams H., Stiff G., Jenkinson L.R., Rees D.I.: Evaluation of the Modified Alvarado Score in acute appendicitis. JR Soc Med 1992; 85:87-88.

17. Adams D.H., Fime C. \& Brooks D. High resolution real time U/S.

18. A new tool in the diagnosis of acute appendicitis. Am. J. Surg. Vol. 155, Jan, 1988.

19. Ohmann C., Yang Q., Frank C.: Diagnostic scores for acute appendicitis. Abdominal pain study group. Eur J Surg 1995; 161; 273-281.

20. Fenyo G., Lindberg G., Blind P., Enochsson L., Oberg A.; Diagnosis decision support in suspected acute appendicitis; validation of a simplified scoring system Eur J Surg 1997; 163 (11); 831-838.

21. Teicher I., Landa B., Cohen M., Kabnick L.S. Wise L.: Scoring system to aid in diagnosis of appendicitis. Ann Surg 1983; 198; 753- 759 .

22. Macklin CP, Radcliffe GS, Merei JM, Stringer MD,: A prospective Evaluation of the Modified Alvarado Score for acute appendicitis in children. Ann R. Coll. Surg. Engl. 1997; 79: 203-205.

23. Sandler, Modified Alvarado Score \& Pearbery J.L. A second approach to the diagnosis of acute appendicitis. The Lancet Vol. 24, 1987, P. 198.

24. Owen TD, William H, Evaluation of the Alvarado score in acute appendicitis. J. R. Soc. Med. 1992; 85: 87-8.

25. Shrivastava UK, Gupta A, Sharma D. Evaluation of the Alvarado score in acute appendicitis. Trop Gastroenterol 2004; 25: 184-6.

26. Sadiq M, Amir, Efficacy of Modified Alvarado scoring system in the diagnosis of acute appendicitis. J postgraduate Med Inst 2002;

27. 16: $72-7$.

28. Stinger J.M., CP Macklin, A prospective study of the evaluation of Modified Alvarado Score for acute appendicitis in children. Ann. R. Coll. Surg. Engl. 1997; 79, 203-205.

29. Russel R.C.G: Williams N.S., Bullstrode C.J.K.; Bailey \& Loves Short Practice of Surgery. 24th edition 2004; $70 ; 1204-10$.

30. Takada T., Yasuda H. \& Shikata J: U/S diagnosis of acute appendicitis complicated by paralytic ileus \& 
generalized peritonitis. J. Clin. U/S, Vol 16, 19988, P. 125-126.

31. Rehman I, Burki T, Alvarado scoring system in the diagnosis of acute appendicitis in children. J Med Sci 2003; 11: 37-41.

32. Gilmore OJA, Jones D, Yang Q. Appendicitis \& mimicking conditions. Lancet 1975; 11: 421-1.

33. Anonymous. A sound approach to the diagnosis of acute appendicitis (editorial). Lancet 1987: 1: 198-200.

34. Fenyo G: Routine use of a scoring system for decision- making in suspected acute appendicitis in adults. Acta Chir Scand 1987; 153: 545-551.

35. Arian GM, Sohukm, Ahmed E, Haider W, Naqi S A. Role of Alvarado score in the diagnosis of acute appendicitis. Pak J Surg 2001; 17:41-6.

36. 33-Dado G. Anania G, Baccarani U, Marcotti E Donini A, Risaliti A et al. Application of a clinical score for the diagnosis of acute appendicitis in children. J Pediatr. Surg. 2000; 35: 1320-2.

37. Smithy W.B., Wexner SD \& Daily T.H: The diagnosis \& treatment of acute appendicitis in the aged. Dis. Col. \& Rec. 1986, P. 170.

38. Safwan A. Taha: Simple ligation versus purse string invagination of the appendix stump in appendicectomy. A prospective randomized study. BJS, Vol. 1 No.2 1995. P. 14-18. 